\title{
Das Eigentliche des Todes. Ein Beitrag zur Be-Lebung der Debatte über Hirntod und Transplantation
}

\author{
Christian Erk
}

Eingegangen: 18. Juni 2012 / Angenommen: 19. Dezember 2012 / Online publiziert: 20. März 2013

(C) Springer-Verlag Berlin Heidelberg 2013

Zusammenfassung Der vorliegende Artikel plädiert dafür, die meist auf die Aspekte Todesdefinition, -kriterium und -feststellung fokussierte Diskussion über die moralische (Un-)Zulässigkeit der Spende und Transplantation vitaler Organe aufzubrechen und beim Nachdenken darüber das zum Ausgangspunkt der Überlegungen zu machen, was mit dem Tod eigentlich verloren geht, nämlich das Leben. Nach einer Antwort auf die Frage „Was ist Leben?" suchend wird hierbei aufgezeigt, dass Leben nicht auf das Vorhandensein gewisser beobachtbarer physiologischer Größen reduzierbar ist, sondern in seinem wesentlichen Kern nur mit den Mitteln der Philosophie als der actus primus alles Lebendigen und das, was wir gemeinhin Seele nennen, erklärt werden kann. Auf Basis der so gewonnenen Erkenntnisse wird geschlussfolgert, dass die Entnahme vitalen organischen Materials aus einer Person, deren Gehirnaktivitäten ausgefallen sind, immer prämortal erfolgt und damit einen Verstoß gegen die Tote-Spender-Regel bedeutet.

Schlüsselwörter Tod $\cdot$ Sterben $\cdot$ Leben $\cdot$ Organspende $\cdot$ Transplantation $\cdot$ Seele

\section{The heart of death. Re-animating the debate on brain death and transplantation}

\begin{abstract}
Definition of the problem Contemporary debates on the moral permissibility of the donation and transplantation of vital organic material often focus on the definition of and criteria for the determination of death. However, death cannot be experienced in the same fashion as life as it is its contradictory negation; death is not-life. Any attempt to define death must, therefore, proceed from and be based on a definition of life. Arguments This article argues that life cannot be reduced to a set of empirically observable properties or functions but can only be explained comprehensively from a philosophical
\end{abstract}

Prof. Dr. phil. C. Erk $(\bowtie)$

Assistenzprofessor für Management und Ethik, Institut für Wirtschaftsethik,

Universität St. Gallen, Tannenstrasse 19,

9000 St. Gallen, Schweiz

E-Mail: christian.erk@unisg.ch 
perspective. Taking this perspective allows us to establish that life is the "actus primus" (soul) of all animate beings. Conclusion Applying this "soul criterion of death" necessitates the conclusion that the explantation of vital organic material from a person whose brain has stopped functioning is always a violation of the dead-donor rule as the person is dying but not dead.

Keywords Death · Dying $\cdot$ Life $\cdot$ Organ donation · Transplantation · Soul

„Der Tod, das unausweichliche Ende von allem, stellte sich ihm zum ersten Mal mit unwiderstehlicher Kraft vor. [...] Er war in ihm selbst - das fühlte er. Wenn nicht jetzt, dann morgen, wenn nicht morgen, dann in dreißig Jahren, war das nicht alles gleich? Und was war dieser unausweichliche Tod, - er wußte es nicht nur nicht, dachte nicht nur niemals auch nur daran, sondern konnte und wagte nicht, daran zu denken. " (Lew N. Tolstoi, Anna Karenina, Dritter Teil, Kapitel 31)

Die Deutschen sind spendeavers - zumindest, wenn es um ihre Organe geht. Um das Angebot an Spenderorganen der Nachfrage anzunähern, besteht seit dem 01. November 2012 das erklärte Ziel des „Gesetzes über die Spende, Entnahme und Übertragung von Organen und Geweben (TPG)“ darin, „die Bereitschaft zur Organspende in Deutschland zu fördern“ (§ 1, Abs. 1 TPG). ${ }^{1}$ Als erster Schritt auf dieses Ziel hin ist die bisher gültige erweiterte Zustimmungsregelung durch die Einfügung von $\S 2$, Abs. 1a TPG durch die sog. Entscheidungsregelung komplementiert worden. Gemäß dieser muss jede in Deutschland krankenversicherte Person, die das 16. Lebensjahr vollendet hat, in regelmäßigem Abstand von ihrer Versicherung aufgefordert werden, ,eine Erklärung zur Organ- und Gewebespende zu dokumentieren“ (§2, Abs. 1a TPG). ${ }^{2}$ Da der hierfür nach $\S 2$, Abs. 1 TPG vorgesehene Organspendeausweis nur eine Erklärung für den Fall zulässt, dass ,nach meinem Tod eine Spende von Organen/Geweben zur Transplantation in Frage kommt", bedeutet dies, dass es vor allem um eine Willenserklärung bzgl. der Möglichkeit der postmortalen Transplantation bzw. Totspende geht. Alle Personen ab dem vollendeten 16. Lebensjahr werden also dazu aufgefordert, sich zu entscheiden und in geeigneter Form festzuhalten, ob sie im Falle ihres Todes, der gemäß $\S 3$, Abs.2, Satz 2 TPG durch die Feststellung des endgültigen, nicht behebbaren Ausfalls der Gesamtfunktion des Großhirns, des Kleinhirns und des Hirnstamms (gemeinhin bezeichnet als „Hirntod“) als eingetreten gilt, ihre Organe zur Transplantation freigeben oder nicht.

Wenn man annimmt, dass sinnvollerweise nur solchen Handlungen zugestimmt werden kann, die man für moralisch zulässig (oder zumindest: nicht moralisch unzulässig) erachtet, geht der Abgabe einer solchen Willenserklärung notwendigerweise die moralische Beurteilung der Handlung voraus, zu der sich erklärt werden soll. Ist diese moralisch zulässig, kann ihr zugestimmt werden; ist sie es nicht, muss die Willenserklärung negativ ausfallen. Die moralische Zulässigkeit der Transplantation von organischem Material (Zellen, Gewebe, Organe, Organsysteme) eines hirntoten Spenders in einen lebenden Empfänger hängt hierbei in erster Linie davon ab, ob die zur Transplantation notwendige Explantation, also die

\footnotetext{
${ }^{1}$ Diese Anpassung des TPG sowie diverse andere sind auf Basis des „Gesetzes zur Regelung der Entscheidungslösung im Transplantationsgesetz" vom 12. Juli 2012 erfolgt.

2 Zu einer Erklärung gezwungen werden kann jedoch niemand (§2, Abs. 2a TPG). Eine Entnahme von Organen oder Geweben ist wie bisher nur erlaubt, wenn der Organ- oder Gewebespender in die Entnahme eingewilligt hatte ( $\$ 3$, Abs. 1, Satz 1 sowie $\S 8$, Abs. 1, Satz 1, lit. b TPG). Liegt im Falle der postmortalen Organentnahme keine Willenserklärung vor, so sind wie bisher die Angehörigen zu befragen (§4, Abs. 1 TPG); eine prämortale Organentnahme ist ohne Willenserklärung des Spenders nicht möglich.
} 


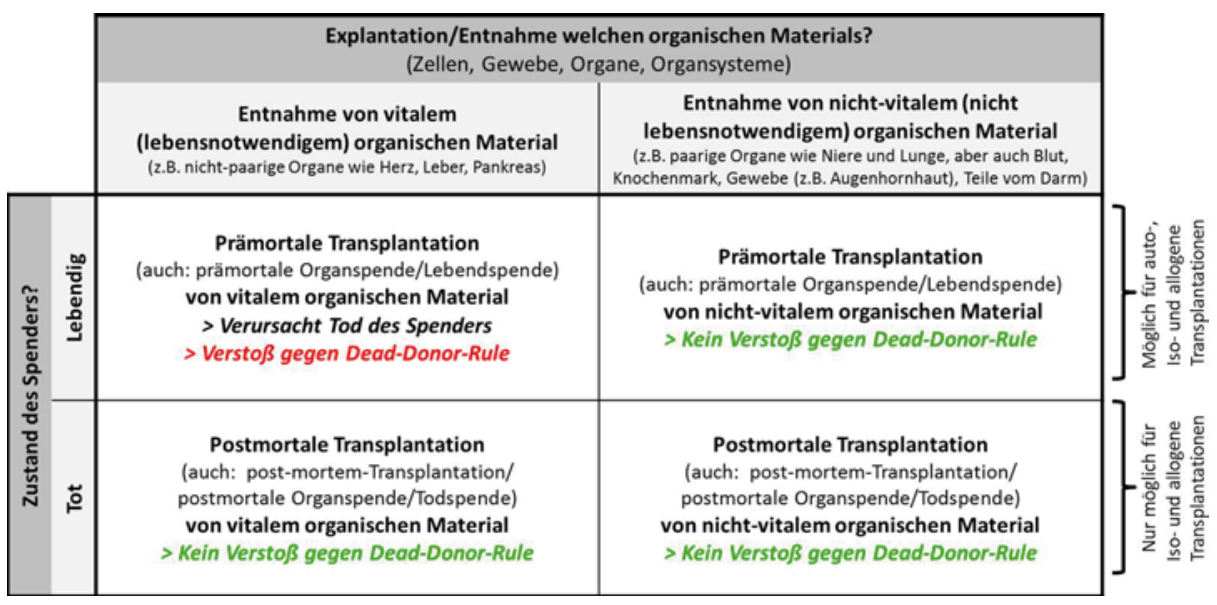

Abb. 1: Formen der Transplantation und Dead-Donor-Rule

Entnahme des zur Transplantation vorgesehenen organischen Materials aus dem hirntoten Spender, einen moralisch zulässigen Akt darstellt oder nicht.

Als Maßstab für die Beurteilung der moralischen Qualität dieser Handlung selbst sollen hierbei die moralische Pflicht, unschuldige Personen nicht zu töten, und das aus dieser abgeleitete moralische Recht auf Leben gelten. Dieses moralische Recht ist nicht nur in Art. 2 Abs. 2 des deutschen Grundgesetzes als positives Recht festgeschrieben (und liegt damit auch dem TPG zugrunde), sondern hat auch als sog. „Dead-Donor-Rule“ (Tote-SpenderRegel $)^{3}$ weltweite Akzeptanz ${ }^{4}$ gefunden. Wie Abb. 1 verdeutlicht, verbietet die Tote-Spender-Regel die prämortale Entnahme von vitalem, d.h. lebensnotwendigem, organischen Material zum Zwecke der Transplantation. Alle anderen dargestellten Formen der Transplantation werden von ihr nicht eingeschränkt, da diese üblicherweise nicht zwingend zum Tod des Spenders führen, sondern allenfalls mit gewissen gesundheitlichen Nachteilen für den Spender verbunden sind.

\section{Die Tote-Spender-Regel und die Explantation von vitalem organischen Material aus Hirntoten}

Wenn wir uns Gedanken darüber machen, ob der Transplantation von vitalem organischen Material eines hirntoten Spenders in einen lebenden Empfänger als moralisch zulässige

\footnotetext{
${ }^{3}$ Linus Geisler formuliert die Tote-Spender-Regel wie folgt: „Lebenswichtige Organe dürfen nur von toten Patienten entnommen werden; lebende Patienten dürfen nicht für oder durch eine Organentnahme getötet werden" ([15], S.4). Vgl. auch ([37], S. 674).

${ }^{4}$ In diesem Zusammenhang ist der Vollständigkeit halber anzumerken, dass es durchaus Stimmen gibt, die dafür plädieren, von der Tote-Spender-Regel abzurücken. So ist es z. B. für Robert Troug und Franklin Miller [37] moralisch nicht relevant, wann eine Person tot ist oder ob sie durch die Organentnahme stirbt, da sie ihr Recht auf Leben, wenn sie einmal in den Sterbeprozess eingetreten ist, freiwillig autonom durch informierte Zustimmung aufgeben kann. Ebenfalls für die Aufgabe dieser Regel (zusammen mit der Vorstellung der Heiligkeit des Lebens), allerdings auf Basis eines an Lebensqualität orientierten utilitaristischen Arguments, sprechen sich Peter Singer ([24], S. 20 ff.) und Dieter Birnbacher [6] aus. Diese Argumente können hier jedoch nicht näher besprochen werden.
} 
Handlung zugestimmt werden kann, dann ist im Grunde zu fragen, ob es sich hierbei um eine prämortale Transplantation von vitalem organischen Material handelt oder nicht. Wenn ja, kann auf dem Organspendeausweis nur das Feld „Nein, ich widerspreche einer Entnahme von Organen und Geweben" angekreuzt werden. Eine Antwort auf diese Frage ist hierbei als Konklusion des folgenden Arguments zu sehen:

- Prämisse 1 (P1): Die Entnahme vitalen, d. h. lebensnotwendigen, organischen Materials (Zellen, Gewebe, Organe, Organsysteme) zum Zwecke der Transplantation ist gemäß der Tote-Spender-Regel moralisch nur zulässig, wenn die Person P, der das organische Material entnommen wird, tot ist.

- Prämisse 2 (P2): Eine Person P ist tot, wenn ...

- Konklusionen (C)

- (C1): Eine Person P, deren Gehirnfunktionen irreversibel ausgefallen sind, ist tot $<>$ nicht tot.

- (C2): Die Entnahme vitalen organischen Materials von einer Person P, deren Gehirnfunktionen irreversibel ausgefallen sind, zum Zwecke der Transplantation ist somit moralisch zulässig<> moralisch nicht zulässig.

Wie aus diesem Argument ersichtlich, hängen die beiden Konklusionen wesentlich davon ab, was als P2 vorausgesetzt wird, was also Tod ist.

\section{Was und wann ist Tod? Todesdefinition, Todeskriterium, Todesfeststellung und das Eigentliche des Todes}

Die Frage, (ab) wann jemand tot ist, ist die Frage danach, woran erkannt werden kann, dass jemand tot ist. Um die unvollständig formulierte Prämisse P2 komplettieren zu können, bedarf es also der Definition eines Todeskriteriums, d.h. eines Kriteriums bzw. Sachverhalts, das den Todeseintritt markiert und anhand dessen sicher festgestellt werden kann, ob jemand die Grenze zwischen Leben und Tod unumkehrbar überschritten hat. ${ }^{5}$

Um eine Aussage darüber machen zu können, wann etwas der Fall ist, muss aber logischerweise zuerst gewusst werden, was überhaupt der Fall sein soll. Um die Frage nach dem Todeskriterium (,Wann ist eine Person P tot?") sinnvoll beantworten zu können, bedarf es also der vorgängigen Beantwortung der Frage „Was ist Tod?“. Ein Todeskriterium setzt also notwendigerweise eine Todesdefinition voraus (Abb. 2).

Was also ist das, „Tod“"? Wenn wir hierüber nachzudenken beginnen, kommen wir schnell an einen Punkt, an dem wir merken, dass es praktisch nicht möglich ist, diese Frage in positiver Form zu beantworten. Wir können zwar sagen, dass der Tod das einzig Sichere in unserem irdischen Leben ist (,sola mors certa est“" [3], Sermo 97, 3)6; wir können auch sagen, dass der Tod am Ende eines Prozesses steht, den wir gemeinhin als „Sterben“ bezeichnen - ein Prozess, den wir alle durchlaufen müssen, wenn auch jeweils auf unterschiedlichen Wegen und in unterschiedlicher zeitlicher Ausdehnung. Doch ist damit noch nichts über das

\footnotetext{
${ }^{5}$ Da ein solches Todeskriterium nur sinnvoll ist, wenn und insofern es auch praktisch handhabbar ist, bedarf es des Weiteren der Definition medizinisch-diagnostischer Testverfahren und Parameter, anhand derer sich der Tod, d.h. die Erfüllung des entsprechenden Todeskriteriums, auch tatsächlich feststellen lässt.

${ }^{6}$ Vgl. auch: „Quid enim in hac terra certum est, nisi mors? [...] incerta omnia: sola mors certa“([4], In Psalmum 38, 19).
} 


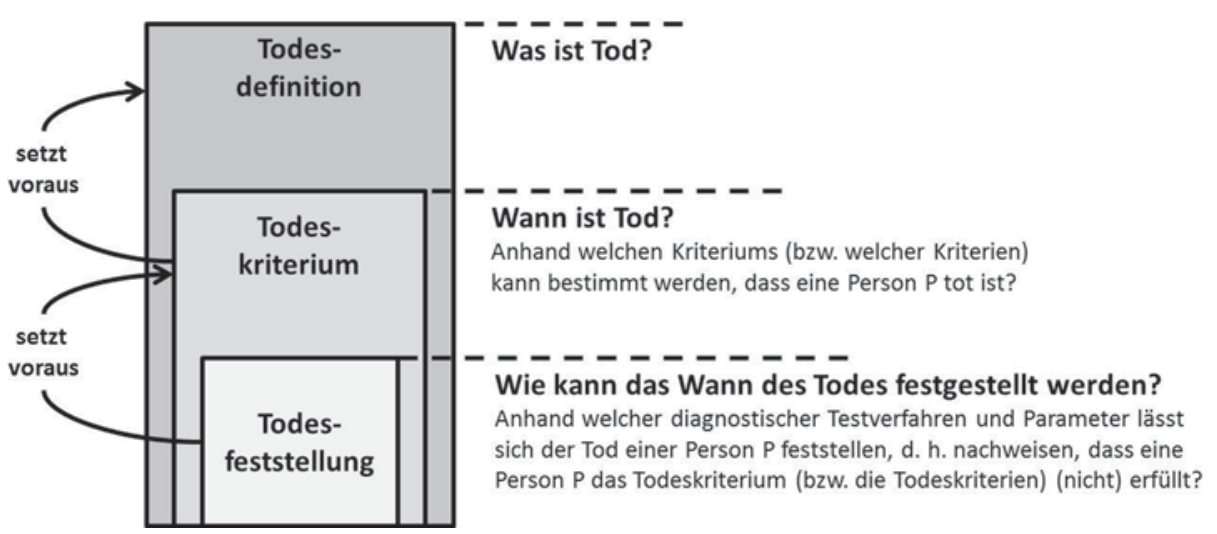

Abb. 2: Todeskriterium und -feststellung setzen eine Todesdefinition voraus

Eigentliche des Todes ausgesagt - und ich wage zu behaupten, dass auf direktem, positivem Wege auch nichts Wesentliches über den Tod ausgesagt werden kann. Da der Tod als Zustand (im Gegensatz zum Prozess des Sterbens) unserer Erfahrung entzogen ist (bzw. wir unsere Erfahrung niemandem mehr mitteilen können (vgl. [19], S.23), können wir leichter sagen, was der Tod nicht ist, als was er ist. So paradox es vielleicht klingen mag: Um etwas über den Tod zu erfahren, müssen wir den Umweg über das nehmen, was er nicht ist, was seine Negation ist, was in kontradiktorischem Widerspruch zu ihm steht ${ }^{7}$ - und das ist das, was wir gemeinhin „Leben“ nennen.

Die Frage „Was ist Tod?“ kann und muss in einem ersten Schritt also schlicht und einfach beantwortet werden mit: Tod ist die Abwesenheit von Leben. Eine grundlegende Einsicht in das Eigentliche des Todes besteht somit darin, dass das Sterben der sich vollziehende Prozess und der Zustand des Todes der vollzogene, abgeschlossene Prozess des Verlustes des Lebens ist. Im Tod haben wir nicht primär irgendwelche Gehirnströme, die Integrität unseres Organismus oder unsere Herz-Lungentätigkeit verloren, sondern unser Leben. Jede weitere Aussage über den Tod ist davon abhängig, was wir unter „lebendig“ bzw. „Leben“ verstehen. Die Todesdefinition lautet in allgemeingültiger Form also: Der Tod ist der abgeschlossene und irreversible Verlust des Lebens.

Die übliche Unterscheidung zwischen Todesdefinition, Todeskriterium und Todesfeststellung muss somit zwingend noch um den Punkt „Lebensdefinition“ ergänzt werden. Wie Abb. 3 darlegt, sind die Kriterien zur Todesfeststellung nur so gut wie das ihnen zugrunde liegende Todeskriterium, das Todeskriterium nur so sinnvoll wie die Todesdefinition, von der es abgeleitet ist, und die Todesdefinition nur so haltbar wie die Definition von Leben, deren negative Formulierung es ist.

Vor dem Hintergrund dieser Überlegungen wird ersichtlich, dass die Prämisse P2 des oben formulierten Arguments, die den Aspekt Todesfeststellung betrifft (was durch eine Umformulierung verdeutlicht wird), nicht ohne eine vorgängige Beschäftigung mit den Aspekten Todeskriterium, Todesdefinition und Lebensdefinition vervollständigt werden kann:

\footnotetext{
${ }_{7}$ Oder wie der hl. Albertus Magnus es ausdrückt: „,mors vitae opponitur privative“ ([1], S. 348 (Tractatus I, Caput III))
} 

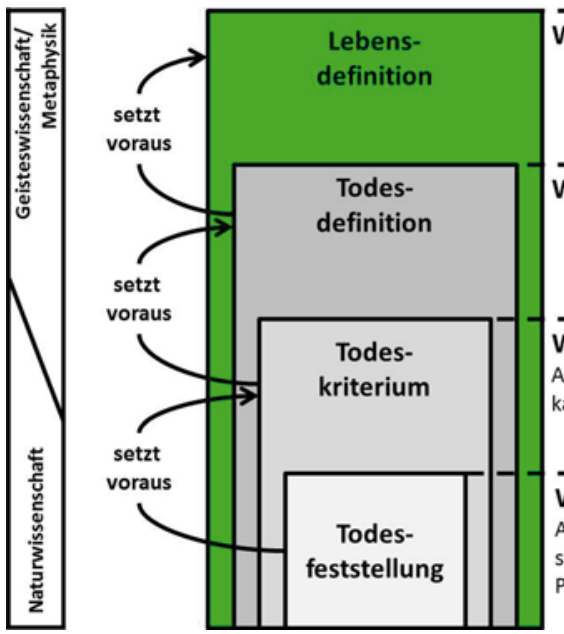

Was ist Leben?

$\overline{\text { Was ist Tod? }}$

Wann ist Tod?

Anhand welchen Kriteriums (bzw. welcher Kriterien)

kann bestimmt werden, dass eine Person $\mathrm{P}$ tot ist?

\section{Wie kann das Wann des Todes festgestellt werden?}

Anhand welcher diagnostischer Testverfahren und Parameter lässt

sich der Tod einer Person P feststellen, d. h. nachweisen, dass eine

Person P das Todeskriterium (bzw. die Todeskriterien) (nicht) erfüllt?

Abb. 3: Lebensdefinition als Voraussetzung für Todesdefinition, -kriterium und -feststellung

- Prämisse 2 (=Todesfeststellung): Der Tod (=Verlust des Lebens) einer Person P gilt als nachweislich sicher festgestellt, wenn...

Die Antwort hierauf ist abhängig vom zugrunde gelegten Todeskriterium:

- Todeskriterium: Eine Person P ist tot (=lebt nicht mehr), wenn...

Die Antwort hierauf ist abhängig von der zugrunde gelegten Todesdefinition:

- Todesdefinition: Der Tod ist der abgeschlossene und irreversible Verlust des Lebens. Eine Person P ist tot, wenn sie nicht mehr lebt.

Die Antwort hierauf ist abhängig von der zugrunde gelegten Lebensdefinition:

- Lebensdefinition: Leben ist ...

Da wir uns also nicht nur über unser persönliches Leben, sondern grundsätzlich nur über das Leben dem Tod annähern können, müssen wir uns zunächst Gedanken über die Frage „Was ist Leben?“ - „die Grundfrage, die allen Fragen zugrunde liegt“ ([8], S. 7) - machen. Im Rahmen der Debatte über die moralische Zulässigkeit der Entnahme vitalen organischen Materials aus Hirntoten tut somit nicht so sehr die Diskussion der Frage not, ob der Hirntod für sich betrachtet ein sinnvolles Todeskriterium ist, sondern welche Vorstellung von Leben diesem Konzept zugrunde liegt und ob diese sinnvoll ist.

\section{Was ist Leben? Die Antwort der Biologie (Naturwissenschaft)}

Üblicherweise wird die Beantwortung der Frage „Was ist Leben?“ der Kompetenz der Naturwissenschaft und hier insbesondere der Biologie als Naturwissenschaft des Lebens zugesprochen. Wenn wir die Biologie bzw. aktuelle Lehrbücher der Biologie dazu befragen, was sie uns bzgl. des Was des Lebens sagen, so finden wir z. B. folgende Antworten: 
- „Wenn es auch nicht möglich ist, Leben wissenschaftlich exakt zu beschreiben, so können wir es doch an seinen Funktionen erkennen: an Stoffwechsel, Wachstum, Bewegung, Vermehrung und Vererbung“" ([9], S.4).

- „Leben ist eine organisierte genetische Einheit, die zu Stoffwechsel, Fortpflanzung und Evolution fähig ist" ([22], S.2).

- „Wir erkennen das Leben an dem, was Lebewesen tun“ ([10], S.3).

Wie aus diesen Aussagen ersichtlich wird, besteht die Antwort der Biologie auf die Frage „Was ist Leben?“ im Hinweis darauf, dass Leben sich in diversen empirisch beobacht- und messbaren charakteristischen Funktionen bzw. Fähigkeiten äußert; ein Objekt lebt, wenn sich an ihm die Fähigkeit $F$ bzw. die Fähigkeiten $F_{1}$ bis $F_{n}$ nachweisen lassen. Die Liste der zu einer solchen Beschreibung des Lebens herangezogenen Merkmale umfasst üblicherweise die folgenden Funktionen bzw. Fähigkeiten (vgl. hierzu stellvertretend [17]; [8], S. 51-70; [22], S. 2 f.; [10], S. 4; [9], S. 4 sowie [18], S. 1 ff.):

- Motilität (selbständiges/aktives Bewegungsvermögen)

- Stoffwechsel (Stoff- und Energieumwandlung, Metabolismus)

- Reproduktion (Fortpflanzung, Vermehrung, Vererbung)

- Regeneration, Wachstum und Entwicklung

- Irritabilität (Erregbarkeit bzw. Reizbarkeit, inkl. Informationsaufnahme, -verarbeitung)

- Kommunikation

- Anpassungsfähigkeit, Reaktion, Regulation

- Ordnung

- Aufbau aus Zellen

- Autopoiese

- ...

Wie durch die Pünktchen beim letzten Aufzählungszeichen angedeutet, ist diese Zusammenstellung nicht als abschließend zu betrachten; so könnte man diese Liste z. B. noch um das implizit aus dem Hirntodkonzept ableitbare Merkmal „Nachweisbarkeit von Gehirnströmen“ ergänzen. Der Umfang der Liste und selbst die genauen Begrifflichkeiten, die von Autor zu Autor variieren, sind jedoch für die Zwecke dieses Artikels nicht weiter relevant.

Während eine solche Liste auf den ersten Blick ein konzises Bild davon zu zeichnen scheint, was Leben ist, so enthüllt ein genauer Blick ein schwerwiegendes Problem: Nicht alle durch Aufnahme in obige Liste als Kennzeichen des Lebens postulierten Merkmale lassen sich bei allen Wesen, die wir als lebendig erachten, nachweisen. So haben z. B. Kenneth und Janet M. Storey nachgewiesen, dass gewisse polare Fisch-, Frosch- oder Insektenarten Temperaturen von unter null Grad überleben können, ohne Zeichen von Stoffwechsel oder Respiration aufzuweisen (vgl. auch [27], S.368f.):

Hundreds of species of terrestrial insects survive long periods of freezing while they overwinter. At the extreme, insects of the high arctic, such as woolly bear caterpillars [...] may spend 10 months of the year frozen solid at temperatures that descend to $-50^{\circ} \mathrm{C}[\ldots]$ or even lower. [...] But of the greatest interest [...] are a group of amphibians and reptiles that survive freezing during their winter hibernation. [...] While frozen, all these animals show no movement, respiration, heart beat or blood circulation, and $[\ldots]$ barely detectable neurological activity. ([26], S. 62 f.) 
Würden wir sagen, dass diese Lebewesen im tiefgefrorenen Zustand tot sind und nach dem Auftauen wieder lebendig werden? Wohl eher nicht; unsere Intuition sagt uns, dass sie die ganze Zeit über lebendig sind, auch wenn sich nicht alle Charakteristika des Lebendigen an ihnen nachweisen lassen. Wir können aus der Tatsache, dass sich Leben anhand von einer bestimmten Anzahl (x) von Fähigkeits- bzw. Funktionsmerkmalen empirisch umfassend und erschöpfend beschreiben lässt, nicht automatisch schließen, dass, wenn an einem Lebewesen z. B. nur $\mathrm{x}-1$ Merkmale nachzuweisen sind, dieses Lebewesen nicht lebendig ist.

Der Versuch, aus der umfassenden Liste der Merkmale des Lebens diejenigen Kennzeichen oder dasjenige Kennzeichen herauszuarbeiten, die bzw. das ausnahmslos alle Lebewesen aufweisen, mag zwar das durch die Entdeckungen von Kenneth und Janet M. Storey aufgeworfene Problem lösen, jedoch nur auf Kosten eines Verlusts an Reichhaltigkeit: „,it loses the fullness of the content of life" ([23], S. 38). Wir opfern die wertvollen Einsichten darüber, in wie vielen Facetten sich Leben äußern kann, einem Reduktionismus, der sich nicht mehr mit unseren alltäglichen Erfahrungen in Einklang bringen lässt. In dieser Beschränkung auf ein Merkmal des Lebendigen liegt auch das große Irritationspotential des Hirntodkriteriums; denn es ist in der Tat mehr als kontraintuitiv und grenzt für manche an Willkür, Personen nur aufgrund des Ausfalls ihrer Gehirnfunktionen für tot zu erklären, obwohl sie bekanntlich eine Vielzahl anderer Merkmale des Lebens zeigen, d. h. u. a. atmen, schwitzen, Gänsehaut bekommen, weinen und ein Kind zeugen bzw. empfangen können, sich warm anfühlen, einen intakten Kreislauf besitzen, deren Haut heilt, wenn sie verletzt wird, und die für die Entnahme von Organen betäubt werden.

Wenn wir uns also auf die empirisch zugänglichen Merkmale des Lebens fokussieren, stehen wir vor der Wahl, das Phänomen „Leben“ entweder durch eine umfassende Liste an relativen, nicht von allen Lebewesen geteilten Funktionen bzw. Fähigkeiten oder durch eine bzw. wenige absolute, von allen Lebewesen geteilte, aber zwangsweise die Reichhaltigkeit des Phänomen „Lebens“ reduzierende Funktionen bzw. Fähigkeiten zu beschreiben. Wie es aussieht, müssen wir uns also für eine von zwei mangelhaften Alternativen entscheiden. Doch bei genauerem Hinsehen fällt an beiden Alternativen ein Mangel auf, der uns vom Zwang zur Entscheidung entbindet und auf eine grundlegendere Ebene führt, die in der öffentlichen Diskussion leider viel zu selten Berücksichtigung findet. Denn der eigentliche Mangel beider Antworten auf die Frage „Was ist Leben?“ ist, dass die Antworten gar nicht die ihr gestellte, sondern eine ganz andere Frage (nämlich: „Anhand welcher Merkmale lässt sich beobachten, dass etwas lebendig ist?“") beantwortet, ja erstere gar nicht beantworten kann. Diese Unfähigkeit liegt darin begründet, dass der naturwissenschaftliche Erklärungsansatz nicht aus dem Korsett der aus seiner Sicht zulässigen Methoden der Empirie schlüpfen kann und sich auf das Beobachtbare und somit ein im Grunde rein funktionalistisches Verständnis von Leben begrenzen muss. Sich mit der funktionalistischen Erklärung von Leben zu begnügen wäre aber gleichbedeutend damit, sich damit zufriedenzugeben, wenn die Frage „Was ist Wind?" mit „Wind ist, wenn es im Wald rauscht oder die Blätter an den Bäumen wackeln.“ beantwortet wird - doch über das Eigentliche des Windes, darüber, was Wind ist, wäre hiermit noch nichts gesagt. Und wenn wir uns bei der Frage nach dem Wind mit einer solchen Antwort nicht zufriedengeben (da wir wissen, dass wir dabei oberflächliche Phänomene mit der Sache an sich, d. h. der Ursache der Phänomene, verwechseln würden), wieso sollten wir es bei der Frage nach dem Leben? Auf der Ebene des Beobachtbaren lassen sich zwar wichtige Erkenntnisse gewinnen; diese helfen uns jedoch nicht, die gestellte Frage nach dem Eigentlichen des Lebens zu beantworten. Hierfür müssen wir noch einen Schritt weitergehen; und diesen Schritt zu tun hilft uns die Philosophie. 


\section{Was ist Leben? Die Antwort der (aristotelisch-thomistischen) Philosophie}

Was verrät uns also ein Blick durch die Brille der Philosophie, welche auf Weisheit und damit den letzten Grund der Dinge ausgerichtet ist (vgl. [13]), über das, was wir Leben nennen? Aus Sicht der Philosophie (zumindest der aristotelisch-thomistischen) ist Leben der Grund dafür, dass die beobachtbaren Merkmale des Lebens überhaupt zustande kommen. Leben ist das, was die oben beschriebenen funktionalen Merkmale des Lebendigen (Motilität, Stoffwechsel, etc.) erst ermöglicht - Leben ist die Ursache dafür, dass es überhaupt Merkmale des Lebendigen gibt.

Was hierunter genau zu verstehen ist, lässt sich anhand der aristotelisch-thomistischen Unterscheidung zwischen Materie (materia) und Form (forma) sowie derjenigen zwischen Akt (actus) und Potenz (potentia) herausarbeiten. Gemäß dieser ist jedes sinnlich wahrnehmbare Seiende und damit auch jedes konkrete Lebewesen als primäre Substanz ein „hylomorphic compound“ [11], d.h. ein aus Materie und Form bestehendes Kompositum (substantia composita). Materie ist hierbei das Material, der Stoff, aus dem die primäre Substanz besteht, und Form das strukturelle Prinzip bzw. der „Bauplan“, nach dem die Materie zu einem bestimmten Wirklichsein organisiert bzw. geformt wird. Jede substantia composita ist dabei nur dadurch seiend (d.h. ein „ens actu“ [36], lib. 2 1. 1 n. 5), dass sich in ihm Materie und Form miteinander verbunden haben. Es ist hierbei ein Charakteristikum von Materie, dass sie potentiell sein kann, was sie aktuell nicht ist (sie ist Potenz, d. h. Möglichkeit zu etwas oder Mögliches), wobei die in der Materie angelegte Möglichkeit durch die Form nur deswegen in die Wirklichkeit überführt werden kann, da Form etwas bereits Wirkliches (Akt; actus) ist (vgl. [36], lib. 21.1 n. 5 sowie [28], I- q. 76 a. 1 co.). ${ }^{8}$

Wie Aristoteles anhand des Unterschieds zwischen dem Besitz und dem Gebrauch von Wissen erklärt, kann man von actus in zweierlei Hinsicht sprechen bzw. kann Form auf zwei Arten actus sein (vgl. [2], 412a11 sowie 402a25-26; [36], lib. 2 1. 1 n. 6; [36], lib. 21. 1 n. 17 sowie [14], S. 108): Wissen zu besitzen und dieses nicht zu gebrauchen, entspricht

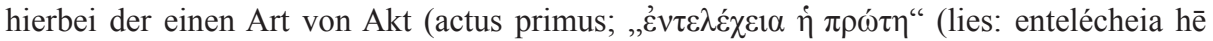
prōtē) ([2], 412a2799)), der Gebrauch dieses Wissens durch z. B. einen Akt des Denkens oder Sprechens der anderen Art (actus secundus). Actus primus entspricht somit einer Fähigkeit,

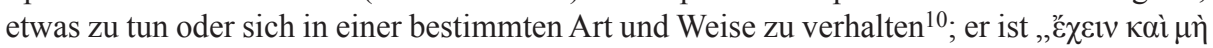

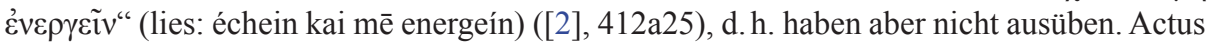
secundus hingegen entspricht der Tat oder dem Verhalten, das sich aus dem Gebrauch dieser Fähigkeit ergibt; er ist die „operatio et usus principii habiti“ ([36], lib. 2 1. 2 n. 7; vgl. auch

\footnotetext{
${ }^{8}$ Der Akt bewegt die Potenz; d.h. das, was im Zustand der Möglichkeit existiert, kann nur von etwas, was bereits im Zustand der Wirklichkeit existiert, in den Zustand der Wirklichkeit überführt werden (vgl. [30], lib. 81.8 n. 1, 2 und 7). In diesem Sinne ist das Wirkliche sowohl der Zeit als auch dem Begriff nach früher als das Mögliche (vgl. [29], lib. 2 cap. 78 n. 10; [36], lib. 21.1 n. 18 sowie [31], lib. 91.7 n. 1).

${ }^{9}$ Die nicht unumstrittene Frage, was genau unter der aristotelischen (und gemeinhin mit Akt übersetzten)

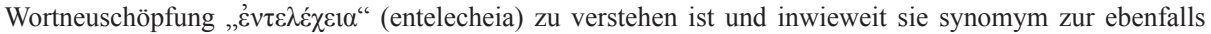

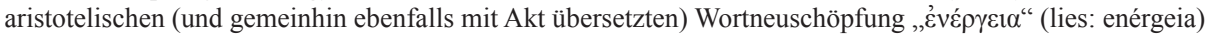

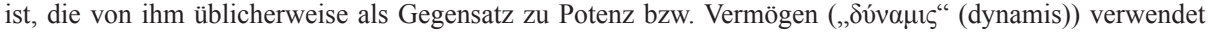
wird, kann und muss an dieser Stelle nicht behandelt werden. Ein Schlüssel zum Verständnis des Begriffs entelecheia, der nicht vollkommen synonym zu energeia zu sehen ist, liegt dabei in der Stelle, in der Aristote-

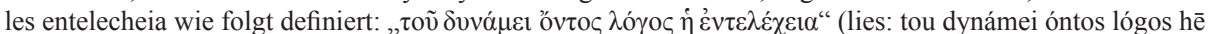
entelécheia) ([2], 415b15). Entelecheia wäre demnach als aktueller Besitz einer Fähigkeit zu sehen, der die Idee bzw. das Ziel ihres potentiellen Gebrauchs (den guten Gebrauch) bereits in sich trägt (actus primus) (vgl. hierzu [23], S. 48 f.), energeia (actus secundus) wäre die konkrete Ausübung der Fähigkeit.

${ }^{10}$ In diesem Sinne ist er auch ,potentia secunda“, also etwas, das aktuell vorhanden ist, von dem aber potentiell Gebrauch gemacht werden kann.
} 
lib. 21.1 n. 19), d.h. die Betätigung und Nutzung eines (operativen) Prinzips, das bereits besessen wird. Die Aussage, dass eine primäre Substanz eine Form (z.B. eine rationale Form) als actus primus besitzt, darf also nicht dahingehend verstanden werden, dass diese primäre Substanz die Fähigkeit zur Rationalität, sondern dass sie Rationalität als Fähigkeit besitzt. Die Fähigkeit, von der im Zusammenhang mit actus primus die Rede ist, ist notwendigerweise immer schon aktuell und wirklich vorhanden - selbst wenn sie nicht genutzt wird oder wenn die Materie, mit der zusammen die Form als actus primus die substantia composita bildet, eine Nutzung nicht oder nicht mehr ermöglicht.

Die spezifische substantielle Form, die nun jeder lebenden substantia composita innewohnt, und dieser ihr Sein und Leben verleiht, bezeichnen Thomas von Aquin und Aristote-

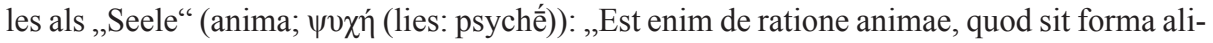
cuius corporis“ ([28], I- q. 75 a. 5 co.; vgl. auch [2], 415b). Die Form „Seele“ zeichnet sich dadurch aus, dass sie der Materie, die sie durchformt, nicht nur Sein verleiht, sondern diese darüber hinaus belebt. Unter Seele ist also die von der Materie als physikalisch-chemischem Aufbau eines körperlichen Lebewesens unterschiedene substantielle Form zu verstehen, die die Ursache dafür ist, dass das betreffende körperliche Lebewesen ist und lebendig ist (vgl. [36], lib. 2 1. 1 n. 15). ${ }^{11}$ Kurz gesagt: Die Seele ist die substantielle Form eines lebendigen Körpers bzw. einer lebendigen substantia composita. ${ }^{12}$

Als Form ist die Seele notwendigerweise auch Akt: ,et hoc est anima, actus, secundum quem corpus vivit" ([36], lib. 21.1 n. 12). Sie ist jedoch nicht einfach actus, sondern wird genauer als actus primus spezifiziert (vgl. [36], lib. 21 . 1 n. 23 sowie [2], 412a27; 412b7). Wenn die Seele actus primus ist, dann ist damit gesagt, dass die Seele nicht als actus ,qui est operatio“ ([36], lib. 21.1 n. 19) zu verstehen ist; vielmehr ist sie das der operatio zugrunde liegende ,principium operationis“ ([36], lib. 2 1. 2 n. 6). ${ }^{13}$ Wenn actus primus dem Besitz und actus secundus dem Gebrauch einer Fähigkeit entspricht, dann bedeutet das, dass mit dem Besitz einer Seele als actus primus gewisse für diese Seele charakteristische Fähig-

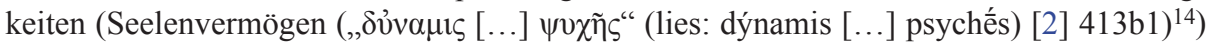
besessen werden. Aristoteles nennt als Beispiele für solche Vermögen einer menschlichen Seele Vernunft, Wahrnehmung, Bewegung und Ernährung (vgl. [2], 413a20). Diese Aussage kann dahingehend verallgemeinert werden, dass durch den Besitz einer Seele ein Lebewesen alle für diese Seele charakteristischen Seelenvermögen, deren Betätigung wir dann als Merkmale des Lebendigen werten, aktuell als Vermögen (d.h. als actus primus) besitzt - unabhängig davon, ob das betreffende Lebewesen von diesen Gebrauch macht

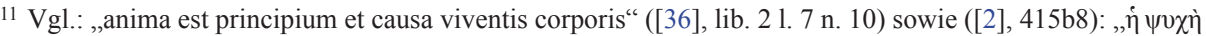

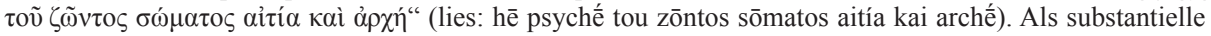
Form (vgl. [36], lib. 21.1 n. 13) und damit auch essentia ist die Seele im Körper aber nicht wie ein Handwerker mit seinem Werkzeug (oder wie Platon es sieht ([21], 115c 2-115e 4) wie der Schiffer mit seinem Schiff) verbunden, d.h. ist nicht nur Wirkursache, sondern „,sie ist in ihm so, wie sich mit dem Bröckchen verflüssigten Silbers die ihm eingeprägte Form [Wappen, Bildnis, Adler] zu der einen, so gestalteten Münze verbindet“ ([19], S. 57. Vgl. auch ([32], cap. 1): ,anima unitur corpori non sicut nauta navi, sed sicut forma.“

${ }^{12} \mathrm{Vgl}$. ([28], I- q. 75 a. 1 co.): „,animata enim viventia dicimus, res vero inanimatas vita carentes.“ sowie

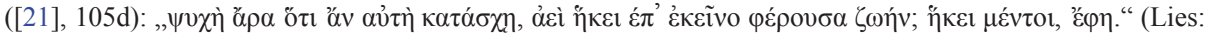
psychế ara hóti av autế katáschē, aeí hếkei ep' ekeíno phérousa zōến? Hếkei méntoi, ephế.) (Siehe hierzu auch [28], I- q. 76 a. 1 co.; [36], lib. 21.3 n. 15; [36], lib. 21.7 n. 11).

${ }^{13} \mathrm{Vgl}$. auch ([36], lib. 21.1 n. 20): „Anima enim, cum sit forma perfectissima inter formas rerum corporalium, est principium diversarum operationum“. Unter „Prinzip“ versteht Thomas von Aquin das, wovon etwas seinen Ausgang nimmt (vgl. [28], Ia q. 33 a. 1 co.) sowie ([31], lib. 5 1. 1 n. 13).

${ }^{14}$ Thomas von Aquin bezeichnet sie als ,operationes animae“ ([36], lib. 21.3 n. 11) und die Seele entsprechend als ,principium operum vitae“ ([36], lib. 21.3 n. 9).
} 
(actus secundus) oder nicht. ${ }^{15}$ Die empirisch beobachtbaren Merkmale des Lebens sind also letzten Endes nichts anderes als actūs secundi bzw. operationes, denen die Form „Seele“ als actus primus bzw. principium operationis zugrunde liegt. ${ }^{16}$

Dass diese Lesart dem entspricht, was Thomas von Aquin und Aristoteles auszusagen im Sinn hatten, wird deutlich, wenn man sich vor Augen führt, dass beide die Seele nicht als Form bzw. actus primus eines lebendigen Körpers, sondern eines Körpers ,habentis in

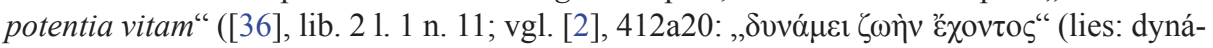
mei zōến échontos)) bezeichnen. Thomas von Aquin und Aristoteles weisen darauf hin, dass sie mit diesem Ausdruck nicht einen Körper bezeichnen, der keine Seele besitzt; vielmehr verstehen sie darunter einen Körper, der eine Seele genannte Form als actus primus besitzt, die damit verbundenen Seelenvermögen jedoch nicht nutzt (vgl. [36], lib. 2 1. 2 n. 6 f. sowie [2], 412b20). Ein beseelter Körper hat also nicht das Vermögen zu leben, sondern besitzt Leben als Vermögen, weil er beseelt ist; damit dies der Fall sein kann, muss Leben aber bereits aktuell vorhanden bzw. besessen sein. ${ }^{17}$ Leben ist also der Besitz von Seele - und damit aller der für die jeweilige Seele charakteristischen Seelenvermögen, deren Gebrauch bzw. Ausübung wir als Lebenszeichen bezeichnen.

Es mag sein, dass die Seele als principium operationis auf operatio hin angelegt ist, Leben sich also in Tätigsein und Aktivität äußert und auf diese hin angelegt ist; jedoch darf es nicht auf diese reduziert werden. Das ist die Einsicht, die wir von Thomas von Aquin und Aristoteles gewinnen können. Auch wenn wir uns einem Verständnis des Phänomens Leben nur über das Sinnenfällige annähern können, so sind Lebenszeichen eben nur Zeichen (Ausdrucksformen) des Lebens, aber nicht das Leben selbst (vgl. [28], I- q. 18 a. 2 co.). Leben ist Haben einer bestimmten Form und damit Sein, ${ }^{18}$ nicht Funktion (vgl. auch [25], S. 168).

\section{Von der Lebensdefinition zum Todeskriterium}

Der Blick durch die Brille der (aristotelisch-thomistischen) Philosophie hat gezeigt, dass Leben als Besitz der substantiellen Form „Seele“ als actus primus und damit als Besitz von Seelenvermögen zu sehen ist, deren Ausübung wir als Lebenszeichen wahrnehmen. Diese Einsicht erlaubt es uns, folgende Definition von Leben zu geben:

- Lebensdefinition: Leben ist Besitz der substantiellen Form „Seele“ als actus primus. Eine Person P lebt, wenn P's Körper die substantielle Form „Seele“ als actus primus besitzt.

\footnotetext{
${ }^{15} \mathrm{Vgl}$. ([28], I - q. 76 a. 1 co.): „Manifestum est autem quod primum quo corpus vivit, est anima. Et cum vita manifestetur secundum diversas operationes in diversis gradibus viventium, id quo primo operamur unumquodque horum operum vitae, est anima, anima enim est primum quo nutrimur, et sentimus, et movemur secundum locum; et similiter quo primo intelligimus. "Wenn dem so ist, ist darin auch die Erklärung dafür zu sehen, warum Aristoteles die Seele nicht energeia, sondern entelecheia nennt, da - wie er im neunten Buch seiner Metaphysik darlegt (1050a 22) - mit energeia Aktivsein bzw. Aktivität bezeichnet werden.

${ }^{16}$ Wichtig ist in diesem Zusammenhang zu betonen, dass die menschliche Seele nicht nur die Form-, Wirkund Finalursache (vgl. [2], $415 \mathrm{~b} 10$ sowie [36], lib. 21.7 n. 10 ff.) ausgewählter Fähigkeiten des Menschen ist, sondern aller menschlichen Fähigkeiten. Für eine Darlegung der durch die menschliche Seele gegebenen Seelenvermögen vgl. ([12], S. 109 ff.).

${ }^{17} \mathrm{Vgl}$. ([36], lib. 2 1. 2 n. 5): ,anima est forma substantialis viventis corporis, et ea remota non remanet corpus vivum nisi aequivoce."

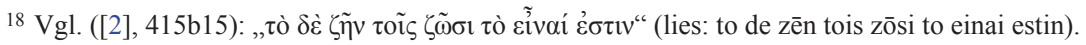


Auf Basis dieser Lebensdefinition und der bereits oben gegebenen Todesdefinition kann nun als nächster Schritt das Todeskriterium abgeleitet werden:

- Todesdefinition: Der Tod ist der abgeschlossene und irreversible Verlust des Lebens. Eine Person P ist tot, wenn sie nicht mehr lebt.

- Todeskriterium: Eine Person P ist tot (=lebt nicht mehr), wenn P's Körper keine Seele mehr besitzt, d. h. wenn sich P's Seele irreversibel von P's bis dahin durch P's Seele beseelten Leib getrennt hat. ${ }^{19}$

Es sei am Rande angemerkt, dass dieses „Seelenkriterium“ des Todes übrigens die von Dieter Birnbacher definierten, aus seiner Sicht universal akzeptierbaren formalen (Qualitäts-) Anforderungen an eine Todesdefinition (vgl. [7], S. 25 ff.) erfüllt und somit auch für diejenigen annehmbar sein sollte, die die dahinter liegende philosophische Position nicht teilen. ${ }^{20}$

\section{Vom Todeskriterium zur Todesfeststellung}

Als letzten Schritt gilt es nun noch, Kriterien zu bestimmen, anhand derer festgestellt werden kann, dass eine Person tot ist, d.h. dass sich ihre Seele von ihrem Leib getrennt hat bzw. wie Thomas von Aquin es ausdrückt: sich aus ihrem Leib zurückgezogen hat bzw. aus diesem gewichen ist (vgl. [36], lib. 21.1 n. 16). Wie lässt sich der Tod also feststellen?

Hierzu ist es hilfreich, sich vor Augen zu führen, dass mit dem Besitz von Seele der Besitz der für diese Seele charakteristischen Seelenvermögen einhergeht, deren Ausübung in beobachtbaren Lebenszeichen resultiert. Dies bedeutet, dass man aus der Tatsache, dass sich an einem Körper gewisse Lebenszeichen beobachten lassen, sicher schließen kann, dass der betreffende Körper ein principium operationis, d.h. eine Seele und damit Leben besitzt, das diese Lebenszeichen ermöglicht. Daraus, dass an einem Körper keine Lebenszeichen beobachtet werden, kann jedoch nicht ohne Weiteres geschlossen werden, dass dieser Körper keine Seele (mehr) besitzt. Von einer Person, die spricht, wissen wir sicher, dass sie Sprachfähigkeit besitzt; von einer Person, die nicht spricht, können wir nicht sicher sagen, ob sie gerade schweigt, aber sprachfähig ist, oder ob sie stumm ist und damit keine

\footnotetext{
${ }^{19} \mathrm{Vgl}$. hierzu auch ([33], lib. 11.15 n. 5.): „per mortem, per quam separatur anima a corpore“; ([21], 64c):

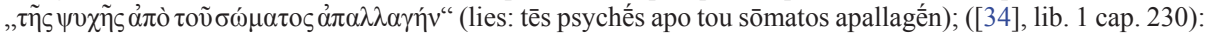
„est de ratione mortis, quod animam a corpore separari“. In diesem Zusammenhang ist es wichtig zu betonen, dass „weder der Leib des Menschen noch seine Seele stirbt, sondern der Mensch selber“ ([19], S. 52) - es stirbt nicht der Leib oder die Seele, sondern der Mensch als „,das aus Leib und Seele gebildete Ganze“ ([19], S. 55). Der Mensch ist nicht nur seine Seele (wie Platon meint (vgl. [20], 129e)), sondern Seele in Leib (,homo non est anima tantum, sed est aliquid compositum ex anima et corpore“ ([28], I $\mathrm{a}$ q. 75 a. 4 co.; vgl. auch [19], S. 56 und 62; ([35], a. 2 ad 5 sowie ([5], XIX, 3)). Laut Thomas von Aquin nimmt in dem Moment, in dem die Seele den Körper verlassen hat, eine andere substantielle Form den Platz der Seele ein (vgl. [36], lib. 2 1. 1 n. 16). Die Materie, die vorher eine bestimmte, seiende und lebende Person war, ist zu etwas anderem geworden, einem Leichnam.

${ }^{20}$ Einzig die Erfüllung von Birnbachers viertem Kriterium, das er allerdings selbst als „umstritten“ ([7], S. 27) bezeichnet, kann diskutiert werden. Nach diesem Kriterium darf laut Birnbacher der Tod stets nur auf das Leben folgen, jedoch nicht das Leben auf den Tod. Wenn Tod und Leben sich als kontradiktorischer Widerspruch gegenüberstehen (wie es sein drittes Kriterium fordert und wie oben herausgearbeitet wurde), dann sind wir, bevor wir ins Leben treten, streng genommen tot; zwischen dem Zustand des Nicht-Seins bzw. Nicht-Lebens und dem des Nicht-mehr-Seins bzw. Nicht-mehr-Lebens besteht aus logischer Sicht kein Unterschied. Somit folgt Leben notwendigerweise immer auf den Tod. Wenn dem so ist, dann ist es unmöglich, dass irgendeine Todesdefinition dieses vierte Kriterium erfüllen kann - was das Kriterium ad absurdum führt.
} 
Sprachfähigkeit besitzt. Um auf die Abwesenheit von Seele und damit Leben schließen zu können, bedarf es somit der grundsätzlichen Unmöglichkeit von Lebensäußerungen, die dann gegeben ist, wenn sich die körperlich-materielle Infrastruktur in einem Zustand befindet, der mit Lebensäußerungen inkompatibel ist bzw. diese verunmöglicht. Der Tod lässt sich also nicht sicher feststellen, wenn die durch den Besitz der Seele gegebenen Seelenvermögen an einem Körper nicht beobachtet werden, sondern nur dann, wenn sie nicht mehr beobachtet werden können, d.h. es unmöglich ist, dass dieser Körper noch Lebensäußerungen zeigen kann.

Da die Seele als actus primus nicht nur ausgewählten, sondern allen beobachtbaren Merkmalen des Lebens zugrunde liegt, bedeutet dies, dass es nicht ausreicht, dass die Äußerung eines oder nur ausgewählter Lebensmerkmale unmöglich ist; vielmehr muss jegliche Lebensäußerung unmöglich sein. Der Tod einer Person P kann also erst dann sicher festgestellt werden, wenn ihr Körper keinerlei wie auch immer geartete Merkmale des Lebendigen zeigen kann. Um den Tod feststellen zu können und zu vermeiden, eine Person nicht vorschnell für tot zu erklären, bedarf es also Kriterien, anhand derer sich sicher bestimmen lässt, dass ein Körper keine Merkmale des Lebens zeigen kann. Die Medizin hat hierzu die sog. sicheren Zeichen des Todes entwickelt. Wenn sich diese an einem Körper nachweisen lassen, kann sicher davon ausgegangen werden, dass der betreffende Körper keine wie auch immer gearteten Merkmale des Lebendigen zeigen kann. Diese Überlegungen erlauben es nun, die Prämisse P2 wie folgt zu komplettieren:

- Prämisse 2 (=Todesfeststellung): Der Tod (=Verlust des Lebens) einer Person P gilt als nachweislich sicher festgestellt, wenn sich an P keinerlei beobachtbare Merkmale des Lebendigen nachweisen lassen und $\mathrm{P}$ in kumulierter Form die unsicheren Todeszeichen ${ }^{21}$ und zudem die frühen sicheren Zeichen des Todes (Leichenflecken (livores mortis) und Leichenstarre (rigor mortis)) $)^{22}$ aufweist. $^{23}$

Wenn diese Bedingungen nicht erfüllt sind, muss davon ausgegangen werden, dass die betreffende Person noch lebt, auch wenn sie gerade stirbt. Wenn wir die so komplettierte Prämisse P2 in das oben dargelegte Argument einsetzen, sind wir in der Lage, die Frage zu beantworten, ob der Entnahme von vitalem organischen Material eines hirntoten Spenders zum Zwecke der Transplantation als moralisch zulässige Handlung zugestimmt werden kann oder nicht.

\footnotetext{
${ }^{21}$ Die unsicheren Todeszeichen (wie z. B. Leichenblässe (palor mortis), Leichenkälte (algor mortis), Atemstillstand, fehlender Puls bzw. Herz-Kreislauf-Stillstand, fehlende Reflexe, Bewusstlosigkeit, Augenhornhauttrübung, Muskulatonie) sind unsicher, da sie zwar sicher post mortem auftreten, aber auch bei lebenden Personen (z. B. bei Vergiftungen) angetroffen werden können.

${ }^{22}$ Von diesen frühen sicheren Todeszeichen, zu denen auch Verletzungen gezählt werden können, die mit dem Leben unvereinbar sind (z. B. Trennung des Kopfes vom Rumpf), werden die späten sicheren Todeszeichen (wie z. B. Fäulnis und Verwesung, Mumifizierung, Besiedelung mit Kleintieren, Skelettierung, Leichenwachsbildung) unterschieden.

${ }^{23}$ An dieser Stelle soll mit einer leider weit verbreiteten Mär aufgeräumt werden, nämlich der, dass sich an (sogar begrabenen) Leichnamen noch Wachstumsprozesse beobachten lassen. Dies ist allerdings nicht der Fall bzw. trügt hier der Schein; die vermeintlichen Wachstumsprozesse von Haaren und Nägeln kommen nicht durch durch Wachstumsprozesse zustande (die ja noch auf vorhandenes Leben schließen lassen würden), sondern sind das Ergebnis von Zersetzungsprozessen des Leichnams. Das Umgebungsgewebe schrumpft und lässt damit Haare und Nägel länger erscheinen: „Alsbald vertrocknen auch Fingerbeeren und Akren, die Konsistenz wird derber, die Farbe rötlich-bräunlich. Diese Vertrocknungen mit ,Verkürzung ${ }^{*}$ der Fingerbeeren bzw. Zehenspitzen lassen die Nägel ,wachsen', d. h. sie wirken aufgrund der Gewebeverkürzung länger als vorher. Dies gilt auch für das, Wachstum " von Barthaaren (der Flüssigkeitsverlust der Gesichtshaut kann zum Hervortreten von Barthaaren führen)“ ([16], S. 119). Vgl. hierzu auch [38].
} 


\section{Konklusion und Schlussgedanken}

Wie die bisherigen Ausführungen deutlich machen, stellt der irreversible Ausfall der Gehirnfunktionen für sich genommen kein hinreichendes Kriterium dar, anhand dessen der Tod einer Person sicher festgestellt werden kann. Hierfür bedarf es der Feststellung, dass jegliche Lebensäußerung unmöglich geworden ist; und dies ist beim Hirntod, bei dem sich eben nur ein Merkmal des Lebens nicht mehr nachweisen lässt, eindeutig nicht der Fall. Wir können also folgende Konklusionen ziehen:

\section{- Konklusionen (C)}

- (C1): Eine Person P, deren Gehirnfunktionen irreversibel ausgefallen sind, ist nicht tot.

- (C2): Die Entnahme vitalen organischen Materials von einer Person P, deren Gehirnfunktionen irreversibel ausgefallen sind, zum Zwecke der Transplantation ist somit moralisch nicht zulässig.

Die Explantation vitaler Organe aus einer Person, deren Gehirnfunktionen irreversibel ausgefallen sind, geschieht somit immer prämortal und tötet darüberhinaus den Spender bzw. beschleunigt dessen Sterbeprozess. Entsprechend kann einer solchen Handlung nicht zugestimmt werden. Personen, die sich wie vom Gesetzgeber angeregt darüber Gedanken machen, wie sie den Organspendeausweis ausfüllen oder ob sie für ihre nächsten Verwandten einer Explantation zustimmen sollen, müssen sich somit bewusst sein, dass sie bzw. ihre nächsten Verwandten, wenn ihnen im Falle des irreversiblen Ausfalls ihrer Gehirnfunktionen vitales organisches Material entnommen wird, nicht nur dieses im Operationssaal lassen, sondern auch ihr Leben. Wenn der Gesetzgeber eine Willenserklärung aller Versicherten fordert, sollte er sie ehrlicherweise auch hierüber aufklären.

Interessenkonflikt Der Autor gibt an, dass keine Interessenkonflikte bestehen.

\section{Literatur}

1. Albertus Magnus (1890) De Morte et Vita. In: Borgnet A (Hrsg) Alberti Magni Opera Omnia, Volumen Nonum: Parvorum Naturalium Pars Prima. Ludovicus Vives, Paris, S 345-372. http://www.archive.org/ details/operaomniaexedit09albe. Zugegriffen: 19. Nov. 2012

2. Aristotle (1907) De Anima. With translation, introduction and notes by R. D. Hicks. Cambridge University Press, Cambridge. http://archive.org/details/aristotledeanima005947mbp. Zugegriffen: 19. Nov. 2012

3. Aurelius Augustinus. Sermones de Scripturis de Novo Testamento (Sermo 51-183). http://www.hsaugsburg.de/ harsch/Chronologia/Lspost05/Augustinus/aug_s000.html. Zugegriffen: 19. Nov. 2012

4. Aurelius Augustinus. Enarrationes in Psalmos. http://www.augustinus.it/latino/esposizioni_salmi/ index2.htm. Zugegriffen: 19. Nov. 2012

5. Aurelius Augustinus. De Civitate Dei. http://www.thelatinlibrary.com/august.html. Zugegriffen: 19. Nov. 2012

6. Birnbacher D (2007) Der Hirntod - eine pragmatische Verteidigung. Jahrb Recht Ethik 15:459-477

7. Birnbacher D (2012) Das Hirntodkriterium in der Krise - Welche Todesdefinition ist angemessen? In: Esser AM, Kersting D, Schäfer CGW (Hrsg) Welchen Tod stirbt der Mensch? Philosophische Kontroversen zur Definition und Bedeutung des Todes. Campus, Frankfurt a. M., S 19-40

8. Brenner A (2009) Leben. Reclam, Stuttgart

9. Buselmaier W (2012) Biologie für Mediziner. 12., neubearbeitete und erweiterte Auflage. Springer, Berlin Heidelberg

10. Campbell NA, Reece JB (2011) Biologie. 8., aktualisierte Auflage. Pearson, München 
11. Cohen MS (2008) Aristotle's metaphysics. The Stanford encyclopedia of philosophy. http://plato.stanford.edu/entries/aristotle-metaphysics. Zugegriffen: 19. Nov. 2012

12. Erk Ch (2011) Health, rights and dignity. Philosophical reflections on an alleged human right. ontos Verlag, Frankfurt a. M.

13. Erk Ch (2012) Verantwortung und Ethik. Z Med Ethik 58:23-36

14. Erk Ch (2012) Potential persons or persons with potential? A Thomistic perspective. Bioethica Forum $5: 105-111$

15. Geisler LS (2010) Die Lebenden und die Toten. Die Transplantationsmedizin beginnt sich von der „Tote-Spender-Regel“ zu verabschieden. Universitas 65:4-13

16. Henssge C, Madea B (2004) Leichenerscheinungen und Todeszeitbestimmung. In: Brinkmann B, Madea B (Hrsg) Handbuch Gerichtliche Medizin, Band I. Springer, Berlin Heidelberg, S 79-225

17. Jessop NM (1988) Theory and problems of zoology. McGraw-Hill, New York

18. Menche N (Hrsg) (2012) Biologie - Anatomie - Physiologie. 7. Auflage. Urban \& Fischer, München

19. Pieper J (1968) Tod und Unsterblichkeit. Kösel-Verlag, München

20. Platon, Alkibiades. http://www.perseus.tufts.edu/hopper/collection?collection=Perseus:collection:Greco-Roman. Zugegriffen: 19. Nov. 2012

21. Platon, Phaidon. http://www.perseus.tufts.edu/hopper/collection?collection=Perseus:collection:GrecoRoman. Zugegriffen: 19. Nov. 2012

22. Purves B, Sadava D, Orians GH, Heller HC (2006) Biologie. Herausgegeben von Jürgen Mark. 7. Auflage. Elsevier, München

23. Seifert J (1997) What is life? The originality, irreducibility and value of life. Rodopi, Amsterdam

24. Singer P (1995) Rethinking life \& death: the collapse of our traditional ethics. Oxford University Press, Oxford

25. Spaemann R (1996) Personen. Versuche über den Unterschied von „etwas“ und ,jemand“. Klett-Cotta, Stuttgart

26. Storey KB, Storey JM (1990) Frozen and alive. Sci Am 12:62-67

27. Storey KB, Storey JM (1996) Natural freezing survival in animals. Ann Rev Ecol Syst 27:365-386

28. Thomas von Aquin. Summa Theologiae. http://www.corpusthomisticum.org/iopera.html. Zugegriffen: 19. Nov. 2012

29. Thomas von Aquin. Summa contra Gentiles. http://www.corpusthomisticum.org/iopera.html. Zugegriffen: 19. Nov. 2012

30. Thomas von Aquin, Commentaria in octo libros Physicorum. http://www.corpusthomisticum.org/ iopera.html. Zugegriffen: 19. Nov. 2012

31. Thomas von Aquin. Sententia libri Metaphysicae. http://www.corpusthomisticum.org/iopera.html. Zugegriffen: 19. Nov. 2012

32. Thomas von Aquin. De unitate intellectus contra Averroistas. http://www.corpusthomisticum.org/ iopera.html. Zugegriffen: 19. Nov. 2012

33. Thomas von Aquin. In librum Aristotelis De generatione et corruptione expositio. http://www.corpusthomisticum.org/iopera.html. Zugegriffen: 19. Nov. 2012

34. Thomas von Aquin. Compendium theologiae ad fratrem Raynaldum. http://www.corpusthomisticum. org/iopera.html. Zugegriffen: 19. Nov. 2012

35. Thomas von Aquin. Quaestio disputata de spiritualibus creaturis. http://www.corpusthomisticum.org/ iopera.html. Zugegriffen: 19. Nov. 2012

36. Thomas von Aquin. Sentencia libri De anima. http://www.corpusthomisticum.org/iopera.html. Zugegriffen: 19. Nov. 2012

37. Truog RD, Miller FG (2008) The dead donor rule and organ transplantation. New Engl J Med 359:674-675

38. Vreeman RC, Carroll AE (2007) Medical myths. Br Med J 335:1288-1289. http://www.bmj.com/content/335/7633/1288. Zugegriffen: 19. Nov. 2012 